\title{
MUJERES, VIDA COTIDIANA Y ESPACIOS PÚBLICOS EN LA REGIÓN METROPOLITANA DE BARCELONA. EL CASO DE CA N'ANGLADA DE TERRASSA
}

\author{
FÀBIA DÍAZ-CORTÉS ${ }^{1}$ \\ MARIA DOLORS GARCIA-RAMON ${ }^{2}$
}

\begin{abstract}
Resumen - El objetivo del artículo es analizar la vida cotidiana a través del uso de espacios públicos por parte de las mujeres residentes en un barrio obrero de la Región Metropolitana de Barcelona - Ca n'Anglada, en la ciudad de Terrassa. La primera parte reflexiona sobre la conceptualización del espacio público desde la perspectiva de género y la segunda parte describe brevemente la metodología utilizada e introduce también el contexto histórico y social del estudio de caso que presentamos. La tercera parte se centra en el estudio empírico de la vida cotidiana de las mujeres en los espacios públicos del barrio escogido. Finalmente elaboramos unas conclusiones y propuestas. La metodología utilizada es cualitativa, básicamente la observación participante y no participante y las entrevistas en profundidad.
\end{abstract}

Palabras clave: Mujeres, espacios públicos urbanos, vida cotidiana, geografía del género, Barcelona.

Resumo - MULHERES, QUOTIDIANO E ESPAÇOS PÚBLICOS NA REGIÃO METROPOLITANA DE BARCElona. O CASO DE CA N'ANGLADA EM TerRassa. O objectivo deste artigo é analisar a vida quotidiana através da utilização de espaços públicos por mulheres residentes num bairro operário da Região Metropolitana de Barcelona, Ca n'Anglada, na cidade de Terrassa. A primeira parte incide sobre a conceptualização do espaço público na perspectiva de género e a segunda descreve sumariamente a metodologia utilizada e introduz o contexto histórico e social do caso em estudo. A terceira parte centra-se na análise empírica da vida quotidiana das mulheres nos espaços públicos do referido bairro. Finalmente, apresentam-se conclusões e algumas propostas. A metodologia qualitativa usada baseia-se na observação participante e não participante e em entrevistas com alguma profundidade.

Palavras-chave: Mulheres, espaços públicos urbanos, quotidiano, geografia do género, Barcelona.

Recebido: 16/12/2009. Aceite: 20/09/2010.

1 Grup d'Estudis de Geografia i Gènere, Departament de Geografia, Universitat Autònoma de Barcelona. E-mail: fabia.diaz@uab.cat

2 Grup d'Estudis de Geografia i Gènere, Departament de Geografia, Universitat Autònoma de Barcelona. E-mail: mariadolors.garcia.ramon@uab.cat 


\begin{abstract}
Women, daily life and public spaces in Barcelona's MetroPOlitAn AREA: A CASE-STUdy of the $C A N$ 'ANGLADA District of TERRASSA. The aim of this article is to discuss the daily use of public space by women in a working class neighbourhood, located within the Barcelona Metropolitan Area: Ca N'Anglada, in the city of Terrassa. Firstly we analyse the concept of public space from a gender perspective secondly we present the research methodology as well as the historical and social context of the case-study. The third part focuses on the empirical analysis of women's daily life and of the use of public spaces in the neighbourhood. Finally, we draw some conclusions and put forth a number of proposals. The research presented in the article made use of qualitative methods, namely participant and non-participant observation, and in-depth interviews.
\end{abstract}

Keywords: Women, public urban space, daily life, geography of gender, Barcelona.

\begin{abstract}
Résumé - LeS FEMMES, LA VIE QUOTIDIENNE ET LES ESPACES PUBLICS DANS LA

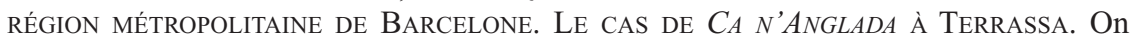
analyse ici la vie quotidienne des femmes d'un quartier ouvrier, à partir de leur utilisation des espaces publics. On réfléchit d'abord sur la notion d'espace public du point de vue du «genre» et on présente brièvement la méthodologie utilisée et le contexte historique et social du cas étudié. Puis la vie quotidienne des femmes dans les espaces publics du quartier est présentée, avant de conclure par des propositions. L'étude, de type qualitatif, est basée sur l'observation participative et non, ainsi que sur des entrevues approfondies.
\end{abstract}

Mots-clés: Femmes, espaces publics urbains, vie quotidienne, géographie du «genre», Barcelone.

\title{
I. INTRODUCCIÓN
}

El propósito de este artículo es analizar la vida cotidiana a través del uso de espacios públicos por parte de las mujeres residentes en un barrio obrero de la Región Metropolitana de Barcelona - Ca n’Anglada, en la ciudad de Terrassa y contribuir de este modo al análisis de situaciones discriminatorias, tanto desde el punto de vista social como espacial, que se dan en muchas ciudades. Las discriminaciones por razones de género son, desgraciadamente, una "normalidad" en nuestra propia cotidianidad y este trabajo las analiza a partir de la cotidianidad en un barrio concreto. El trabajo se ha desarrollado a partir del enfoque de género pero no de forma aislada, ya que la complejidad social y espacial reclama, que se interrelacionen temáticas y aspectos (etnia, clase social, etc.) que van más allá de este enfoque (Hayden, 1995; Ruddick, 1996).

La primera parte reflexiona sobre la conceptualización del espacio público desde diferentes enfoques y disciplinas, incluido el de la geografía del género. La segunda parte describe brevemente la metodología utilizada e introduce también el contexto histórico y social del estudio de caso que presentamos. La tercera parte - la más extensa - se centra en el estudio empírico de la vida 
cotidiana de las mujeres en los espacios públicos del barrio escogido. Finalmente elaboramos unas conclusiones y propuestas.

\section{LOS ESPACIOS PÚBLICOS EN LA CIUDAD Y LA CONTRIBUCIÓN DE LOS ESTUDIOS FEMINISTAS}

El uso y la apropiación de los espacios públicos es un aspecto fundamental en los estudios sobre la vida cotidiana de los hombres y mujeres que viven en la ciudad. Esta experiencia no es la misma para todo el mundo, ya que factores como el sexo, la edad, la clase social y la identidad étnica afectan a la percepción y a la vivencia de la vida urbana (Tello y Quiroz, 2009). Desde una perspectiva sociocultural, los espacios públicos se definen como lugares de interrelación, de encuentro social y de intercambio, donde convergen grupos con intereses diversos (Borja y Muxí, 2001; Borja, 2003). Los espacios públicos pueden definirse como espacios mentalmente abiertos porque suelen diseñarse para una gran variedad de usos, incluso algunos usos que podrían considerarse no deseables (Walzer, 1986; Valentine, 1989; Pain, 1997). Además, cuando son utilizados por una gran variedad de personas y para una gran variedad de actividades, pueden contribuir a la identidad colectiva de la comunidad (Valle del, 1997; Franck y Paxson, 1989).

Desde una perspectiva política, la definición incluiría una visión de los espacios públicos como lugares donde la gente puede participar en la vida pública y donde pueden expresarse los derechos civiles (López de Lucio, 2000). La perspectiva de la arquitectura define los espacios públicos como espacios abiertos y accesibles a todo el mundo, espacios a donde todos pueden ir, en contraste con los espacios privados de acceso restringido (Chelkooff y Thibaud, 1992-1993). Los espacios públicos pueden convertirse en "paisajes participativos", es decir, en elementos centrales de la vida urbana, que reflejan la cultura, las creencias y valores (Francis, 1989). El diseño arquitectónico de un espacio, el mobiliario urbano y la iluminación pueden estimular o desanimar el uso de un determinado espacio por parte de ciertos individuos o grupos. También se ha señalado el papel de los espacios públicos en la integración y la cohesión social y se ha argumentado que la calidad de vida en las calles y en los espacios abiertos es una condición necesaria para que la sociedad alcance un grado satisfactorio de convivencia (Rogers, 1998).

Académicas feministas han señalado el sesgo machista de muchos estudios sobre el medio urbano y el espacio público (Hayden 1981; Greed, 1996; McDowell, 2000). Históricamente, la arquitectura y la planificación urbana, así como su enseñanza y profesionalización, han sido áreas dominadas por hombres. Como resultado de ello ha surgido una visión del espacio urbano homogénea y "desgenerizada" (que no tiene en cuenta la estructura de género de la sociedad), como si los intereses y las necesidades de los hombres fuesen universales (Wilson, 1995). Sin embargo muchas mujeres han reivindicado el derecho a partici- 
par en la planificación y el diseño urbanos a fin de promover una organización no sexista del espacio público (Wekerle, 1984; Bondi, 1998; Sandercock y Forsyth, 2000), recordándose que el diseño de los espacios públicos tiene mayor impacto en las vidas cotidianas de las mujeres que en la de los hombres (Borja, 2003). Esto es debido a que las mujeres tienen una relación con su entorno urbano mucho más estrecha, ya que pasan más tiempo fuera, desarrollando tareas relacionadas con asuntos domésticos o familiares: recogiendo a hijos e hijas del colegio o de actividades extraescolares, haciendo acompañamiento a centros sanitarios o haciendo gestiones relacionadas con la economía doméstica (Prats, 1998; Díaz-Cortés 2006).

Al mismo tiempo, en las sociedades capitalistas el espacio público es también escenario de discriminación y marginación de classe. Y desde las diferentes disciplinas se considera que esta realidad se encuadra en un proceso de mercantilización y privatización del espacio público y, por tanto, de control de las presencias y actividades que se desarrollan en él, haciendo que la vida cotidiana en espacios públicos concretos se base en la capacidad de consumo, en el cierre de clase y/o intracomunitario y la proliferación de espacios de encuentro y consumo custodiados y altamente vigilados (Sennet, 1978; Fernández Durán, 1996; Davis 2001). En este sentido, los procesos sociales de dominación y de resistencia en el espacio público han sido descritos tradicionalmente por la geografía cultural a través del concepto de hegemonía, reforzando interpretaciones de diversas formas de dominación que permiten una mejor comprensión de cómo el poder económico se expresa a través del espacio (Corbridge et al., 1994) y, también, el poder patriarcal, ya que las mujeres continúan padeciendo una situación de discriminación respecto a los hombres. Se destaca que los avances en la ocupación en trabajos remunerados o su presencia en cargos públicos y privados de relieve no ha supuesto un cuestionamiento del patriarcado $\mathrm{y}$, al mismo tiempo ha reforzado las desigualdades de clase (Gleson, 1995; Bondi y Domosh, 1998; Bondi, 1998; Pain y Townshend, 2002; Kern, 2005). Por ello al analizar los usos y la apropiación de los espacios públicos hay que distinguir entre presencias relacionadas con el trabajo doméstico (actividades relacionadas con el hogar y la familia) y otras de carácter más autónomo (Flahault, 2001).

Por ello, la concepción de espacio público como lugar de interacciones sociales y de encuentro aparece cada vez más idílica. Sin embargo, las situaciones en el espacio público son dinámicas, y otros planteamientos subrayan su importancia real y simbólica en la vida cotidiana a través de la espontaneidad (Benach y Tello, 2004; Albet et al., 2006) o la resistencia de colectivos discriminados (Jackson 1989; Greed, 1994; Mitchell, 2003). Es en esta realidad de exclusión y discriminación, pero también de espontaneidad y resistencia, donde el análisis geográfico en barrios y ciudades pone de manifiesto la importancia del papel de las mujeres, tanto desde el punto de vista de colectivo discriminado como de colectivo que no se resigna a las obligaciones o roles que la sociedad patriarcal impone (Vaiou, 2010). 


\section{METODOLOGÍA Y PRESENTACIÓN DEL ÁREA DE ESTUDIO}

Este artículo se basa en el trabajo de campo realizado entre 2002 y 2007 en el barrio estudiado. En la metodología se han combinado métodos cualitativos como la observación no participante y participante y la entrevista cualitativa en profundidad con el trabajo sobre fuentes documentales, estadísticas, gráficas y cartográficas. Las técnicas cualitativas utilizadas se relacionan con una tradición en geografía comprometida con la necesidad de generar un conocimiento que no esté desligado de la experiencia cotidiana de las personas (Taylor y Bogdan, 1996; Ekinsmyth, 2002; Driver, 2003). En este sentido, no se puede pasar por alto que han sido determinados posicionamientos y enfoques en geografía, como las geografías feminista y cultural, que han dado un impulso importante a la geografía de los lugares y de la vida cotidiana (García Ballesteros, 1982 y 1998; Prats, 1997; Crang, 2002 y 2005; Davies y Dwyer, 2007; Garcia Ramon, 2008) y es aquí donde tenemos que situar también este trabajo. La fuente oral la podríamos considerar un importante recurso para poder construir geografías de la cotidianidad y de la memoria (Riley y Harvey, 2007), un aspecto que es reclamado, sobre todo, desde la geografía feminista (McDowell, 1992; Katz, 1994; Hayden, 1995).

En la técnica de observación se ha de distinguir entre una observación no participante, que se ha desarrollado sistemáticamente en un espacio público emblemático del barrio, la Plaza de Ca n'Anglada, y que nos ha permitido elaborar los mapas de uso del espacio de la plaza central del barrio, y la observación participante, que ha supuesto una participación más activa y relacional con las personas. Estos dos tipos de observación han facilitado poder tener en cuenta la diversidad de espacios públicos y de encuentro existentes en el barrio (otras plazas más pequeñas y no céntricas, calles, centro cívico, escuelas, bares, locutorios, tiendas, centros deportivos, etc.), más allá del espacio concreto de la plaza central de Ca n'Anglada. Todas las vivencias que se recogían durante estas observaciones - a través de un bloc de notas y de fotografías - ayudaban a ampliar informaciones y a generar más matices y confianza mutua entre investigadores y vecindario $\mathrm{y}$, también, ejercitar un sentido inherente a la práctica geográfica como es la observación (Driver, 2003).

Si bien la observación ha sido una importante fuente de información, las entrevistas cualitativas en profundidad han supuesto la fuente más densa de información. Se han realizado 49 entrevistas de las que 39 fueron a vecinos y vecinas $^{3}$ y 10 a personal técnico y agentes sociales no residentes en el área de estudio. En todo momento se ha evitado la técnica de "bola de nieve" para conseguir los contactos, y se ha intentado diversificar al máximo las características sociales de las personas entrevistadas. Se grabaron todas las entrevistas y después se codificaron por temáticas que posteriormente se analizaron con la

3 Fueron realizadas individualmente 15 entrevistas a mujeres y 19 a hombres. Se realizaron 4 entrevistas grupalmente, 2 para el caso de mujeres y 2 más para el caso de hombres. Y, finalmente, una entrevista fue mixta, con un hombre y una mujer. 
ayuda del programa informático Atlas-ti, siguiendo las pautas del análisis del discurso (Baylina, 2004; Crang, 2005) ${ }^{4}$.

En cuanto al barrio de estudio seleccionado quisiéramos señalar que $\mathrm{Ca}$ n'Anglada saltó a las primeras páginas de la prensa española y catalana en el verano de 1999 cuando se produjeron lo que podríamos considerar los primeros incidentes violentos organizados y colectivos de carácter racista en España, atacándose a personas de origen marroquí así como a sus bienes y propiedades. El barrio es un ejemplo paradigmático de la evolución de los barrios obreros de la Región Metropolitana de Barcelona en los últimos sesenta años, a los que han ido llegando sucesivos procesos migratorios de referencia desde principios del siglo pasado, en un primer momento originarios de diferentes regiones españolas y a partir de mediados de los años 90 procedentes de fuera de la Unión Europea. Esta superposición de diferentes procesos migratorios no ha estado en absoluto ajena a la creación de conflictos de convivencia que han originado incidentes, a veces violentos, como los de Ca n'Anglada en 1999. En todo caso, no hemos de olvidar que el pasado reciente de barrios como el de Ca n'Anglada son un ejemplo claro de lugares donde las necesidades colectivas contribuyeron a la autoorganización y solidaridad entre vecinos y vecinas dando lugar a la lucha vecinal que supuso una mejora de las condiciones de vida colectivas con la reinstauración de los ayuntamientos democráticos a partir de los años ochenta.

Ca n'Anglada es un barrio que tiene su origen en la periferia de Terrassa (200 000 hab.), una ciudad de fuerte tradición industrial en el contexto de la Región Metropolitana de Barcelona. El barrio tiene sus orígenes en un periodo de fuerte crecimiento migratorio que tiene lugar a partir de la década de 1950. En efecto, entre 1950 y 1970, Barcelona y su área metropolitana crecieron rápida y caóticamente, atrayendo a centenares de miles de inmigrantes de otros lugares de Península Ibérica. La especulación inmobiliaria desenfrenada durante las administraciones municipales franquistas condujo al crecimiento de barrios marginados y de autoconstrucción (como el de Ca n'Anglada) y desencadenó procesos próximos a la fractura social y cultural de estas áreas empobrecidas, que se superó gracias a la autoorganización asociativa del vecindario en interacción con la actividad política y sindical clandestinas desde el antifranquismo. Con la reinstauración de los ayuntamientos democráticos en 1979 se inició un giro en la atención e inversión en este tipo de barrios, suponiendo una clara dignificación, interviniendo en materia de espacios públicos, equipamientos y en servicios públicos y comunitarios (Roca, 1994; Borja, 1995; Busquets, 2004; Montaner, 2004). Y estos cambios fueron posibles gracias a la presión de los movimientos sociales y vecinales que ya se habían originado en los últimos años del franquismo, siendo el caso de Ca n'Anglada paradigmático en este sentido (Lacueva, Márquez y Plans, 2007). No obstante, la existencia de partida de un parque de viviendas precario y que está por debajo de la media general y las características del sector productivo

4 Para un análisis detallado de la metodología véase Díaz-Cortés (2009). 
local y del mercado laboral precarizado en general ha supuesto que este tipo de barrios continúen siendo el foco de asentamiento y concentración de personas y familias con pocos recursos, como veremos a continuación en el caso de $\mathrm{Ca}$ n'Anglada.

El fuerte crecimiento económico español desde 1996 hasta la reciente crisis motivó una segunda y masiva oleada de inmigrantes extracomunitarios en todo el país y, por lo tanto, también en la Región Metropolitana de Barcelona. Estos inmigrantes se fueron asentando en barrios tradicionalmente periféricos y relativamente marginados. Así pues la realidad actual de Ca n'Anglada no está absenta de problemáticas derivadas de un pasado de discriminación e injusticia que todavía hoy son actualidad, pues al igual que ha sucedido en otros barrios de estas mismas características, Ca n'Anglada vuelve a ser refugio de inmigrantes de clase trabajadora que, de nuevo, presentan diferencias culturales.

Entre 1995 y 2006 el barrio pasó de 11924 a 13764 habitantes censados (Ajuntament de Terrassa, 2007), debido, sobre todo, al empadronamiento de personas de origen extracomunitario y al reagrupamiento familiar. Analizando los orígenes principales de la población censada en 2006, se observa que el lugar de nacimiento mayoritario es Cataluña, que representa un 38\% (5 232 personas), y en segundo lugar Marruecos, con un 22\% (3 054 personas). Después se sitúa Andalucía, con un 19\% (2 646 personas) y Ecuador con un 4\% del total (558 personas).

Últimamente se observa un proceso de masculinización que se explica por las características socio-laborales del mercado de trabajo local. En 2007 la tasa de actividad era del $42 \%$ para las mujeres, que trabajan sobre todo en el sector servicios de baja cualificación, y del $66 \%$ para los hombres, que se emplean en le sector industrial, muy en particular en la construcción (Ajuntament de Terrassa, 2007), donde trabajan la gran mayoría de hombres de origen marroquí, sector que ha sufrido en gran manera la crisis actual, que aún no aparece en estos datos.

\section{MUJERES, VIDA COTIDIANA Y ESPACIOS PÚBLICOS EN CA N'ANGLADA DE TERRASSA}

\section{La mujeres de la "antigua" y la "nueva" inmigración en la construcción social del barrio ${ }^{5}$}

El proceso de construcción material del barrio es uno de los principales elementos que ha contribuido a la creación de sentimientos de identidad, de arraigo y pertenencia a Ca n'Anglada de Terrassa. Efectivamente, muchas de las personas que residen en el barrio fueron protagonistas de episodios tan significativos y referenciales como la autoconstrucción de sus casas, la urbanización

5 Denominamos “antigua” inmigración a la que llegó a Ca n'Anglada mayoritariamente entre 1950 y 1970 y "nueva" inmigración a la que ha llegado al barrio a partir de la década de 1990. 
de las calles y la reivindicación de equipamientos e infraestructuras, todo ello combinado con la lucha política contra la dictadura del general Franco. No se trata sólo, pues, de memorias intangibles sino que son elementos identitarios comunitarios que también impregnan muchos lugares y espacios de encuentro del barrio, en especial su plaza central, la Plaza de Ca n'Anglada, donde se desarrolló la observación sistemática no participante. En este sentido, cabe recordar el papel destacado que jugaron las mujeres en la mayoría de estas luchas vecinales, propiciando la formación de esta identidad y arraigo al lugar, que se ha transmitido, en algunos casos, de madres a hijas:

«Yo me considero nacionalista de barrio totalmente, sí, sí, eso sí que lo tengo clarísimo, [...]. Si a mi me preguntan de dónde soy, yo soy de Ca n'Anglada, Terrassa de Ca n'Anglada, sí eso sí. El barrio a mi me ha aportado muchísimo, muchísimo ${ }^{6} \gg$ [28:26] Núria, vecina de Ca n’Anglada, 24 años, nacida en Terrassa. Vive en el barrio desde su nacimiento.

«Por ejemplo el colegio este de aquí fue unas luchas fuertes, hasta con los famosos grises y todo eso. Eran reivindicaciones de barrio, chocolatadas, fiestas, festivales, y eran unas reivindicaciones muy duras y muy llevadas por las mujeres, porqué claro la parte técnica y de control la llevaban las mujeres y no mujeres particularmente de partidos ni nada de eso, no, no, mujeres de asociación de vecinos, del barrio. Porqué había una necesidad imperial». [36:1] Pilar, vecina de Ca n'Anglada, 50 años. Nacida en Terrassa. Vive en el barrio desde los 11 años.

Esto ha sido así hasta el punto que tanto mujeres jóvenes como mayores, establecidas desde hace años en Ca n'Anglada, consciente o inconscientemente, expresan una marcada identidad de barrio que también se extiende sobre espacios públicos concretos que son emblemáticos y referenciales en la historia del barrio, como el caso de la citada plaza central. Los espacios públicos se convierten en lugares sentidos como propios, referencia material en el imaginario colectivo de la población autóctona. Las mujeres recién llegadas a partir de los años 90 , básicamente de origen marroquí y ecuatoriano, no muestran (todavía) un sentimiento similar de identidad, aunque admiten que sus hijos e hijas pueden y deben identificarse con el barrio y la ciudad:

«[...] yo soy de Marruecos y ya está [...], mi marido lleva veinticuatro [años] y que va, igual, aquí se viene a trabajar y ya está. [...] [Mis hijos han] nacido aquí, están contentos, mis hijos están más abiertos porque van al colegio con españoles... [para ellos] bastante fácil, para mi no, ellos han nacido aquí y saben hablar y todo. Cuando me voy a Marruecos quince días y solo quieren que venir aquí... [...]. Yo no me siento de aquí, pero mis hijos sí, ahora uno tiene siete, vamos a Marruecos y no quiere quedarse quiere venir aquí... ellos sí, nosotros no. Porque aquí hay

6 Algunas citas que se exponen en el texto, como la correspondiente a esta nota, fueron expresadas en lengua catalana y se ha optado por traducirlas al castellano para este artículo. 
tanta libertad, todo, el colegio,... ahora se levanta "mama, al cole" corriendo, corriendo, porque cuando entra en el cole hay de todo, en Marruecos no, otra cosa». [19:39 y 19:43] Munira, vecina de Ca n’Anglada, 36 años. Inmigrada recientemente desde Rabat (Marruecos).

De hecho, no sienten suyo el barrio no sólo por no haber vivido directamente este pasado y por la inexistencia de nexos entre el pasado del barrio y las nuevas realidades sociales y culturales, sino porque - sobre todo en el caso de las mujeres de origen marroquí, mayoritarias entre el vecindario recién llegado - se trata mujeres que han perdido gran parte del papel de referencia y predominante que tenían en la vida cotidiana de sus lugares de origen: si allí la mujer era protagonista a la hora de organizar el hogar y la vida familiar, trabajando en el campo y desarrollando múltiples actividades, la vida en Ca n'Anglada de Terrassa pasa a concentrarse (casi exclusivamente) en la vivienda familiar. El marido sale a trabajar o a comprar y los niños, desde muy pequeños, van a la escuela, por lo que trabajo y escuela se convierten en lugares privilegiados para la socialización y la integración de maridos e hijos e hijas, pero no de ellas:

«[...] las madres se quedan en casa, las únicas desfavorecidas aquí son las madres [...]. Y veo que la mujer allí en Marruecos era la protagonista, más o menos, no tanto pero era ella quien gobernaba su casa, trabajaba fuera en el campo, hacía de todo. Aquí pues está en casa, se encierra entre cuatro paredes, y los niños aprenden, el marido se relaciona con la gente y ella no. Y ves que ellas mismas cuando hablo con ellas se sienten un poco angustiadas, y dicen "mi hijo de 5 o 6 años me dice 'mamá cállate, tú no sabes' " por el idioma se siente ella que está perdiendo su papel como madre». [6:5] Amal, personal técnico municipal no residente en el barrio. Inmigrada recientemente desde Ksar-el-Kbir (Marruecos).

La mayoría de los ámbitos controlados por estas mujeres en sus lugares de origen pasan aquí a depender de sus maridos o de terceros (la escuela o la administración municipal) de manera que prácticamente sólo mantienen una influencia clara en los niños y niñas mientras son bebés. Esta situación se agrava por el desconocimiento del catalán o del español (desconocimiento que maridos y, por supuesto, los hijos e hijas superan más rápidamente) y por la falta de socialización (incluso con otras mujeres del mismo origen) que el aislamiento en el hogar conlleva.

\section{Presencias y ausencias en la Plaça de Ca n'Anglada}

\subsection{Las personas usuarias de la plaza}

Las observaciones sistemáticas no participantes, y teniendo en cuenta también las no sistemáticas y participantes, realizadas en diferentes días de la semana y a diferentes horas en la Plaza de Ca n'Anglada, muestran unos usos bien diferenciados según se trate de días laborables o fines de semana (es más utilizada de 
lunes a viernes que en sábado o domingo). Si nos fijamos en franjas horarias por las tardes es cuando hay más afluencia en la plaza, momento en que predomina la presencia de mujeres (madres) y población infantil, mientras que al mediodía el grupo mayoritario con diferencia son los abuelos (ver ejemplo de la fig. 1).

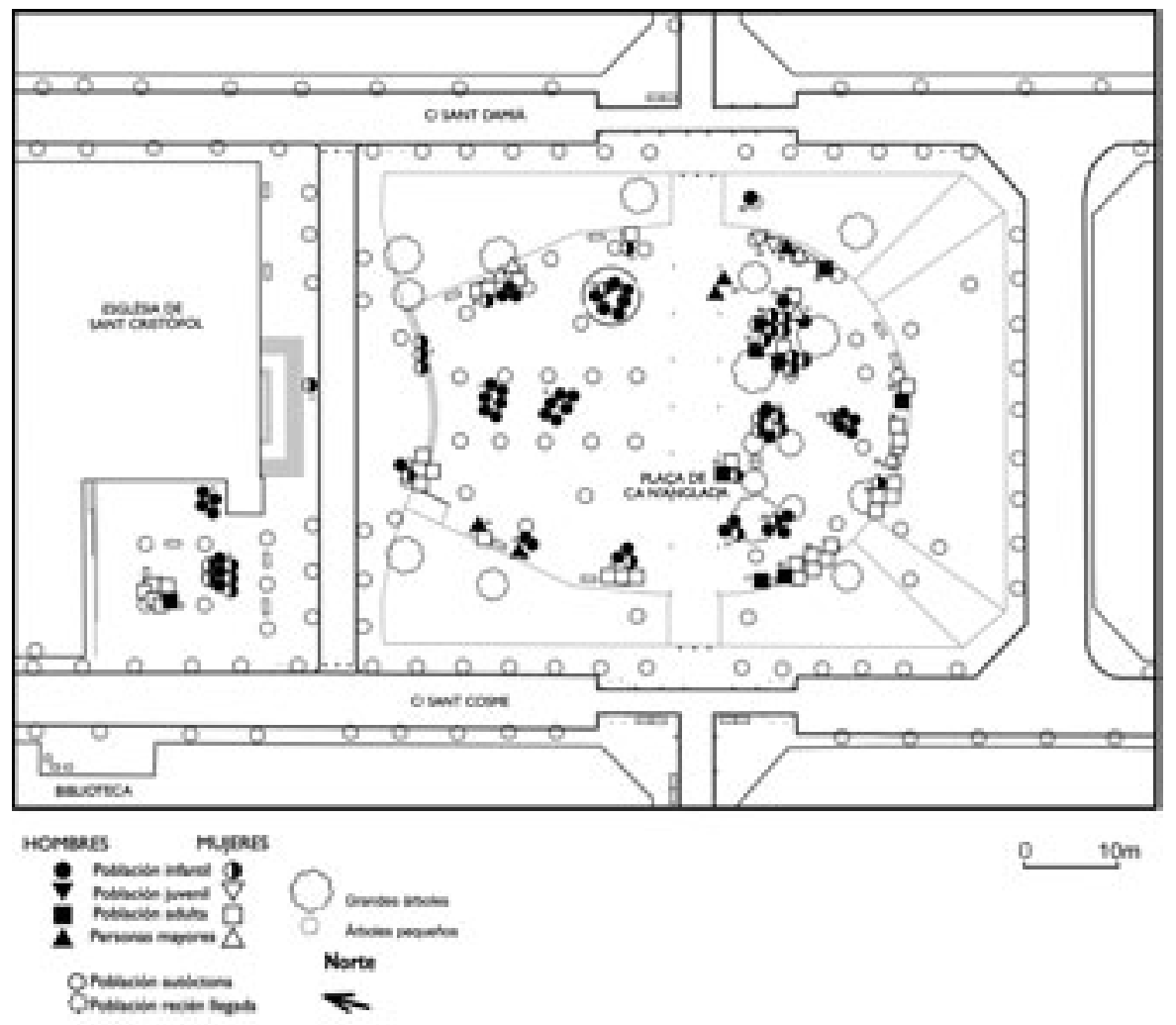

Fuente: Elaboración propia

Fig. 1 - Ejemplo de uso de la Plaça de Ca n'Anglada (martes, 1 de octubre de 2002, 17h:40m-18h:05m).

Fig. 1 - An example of the use of Plaza de Ca n'Anglada (Tuesday, October 1st. 2002, 5h40pm-6h05pm).

Del análisis de la información obtenida en las observaciones (sistemáticas o no) realizadas en la Plaza de Ca n'Anglada destaca, en primer lugar, que el grupo de edad que tiene más presencia es el de los abuelos u hombres mayores jubilados, al mediodía y, el de la población infantil por las tardes. La población juvenil y los hombres adultos tienen una presencia muy marginal. El caso de la mujer adulta destaca por ser un colectivo con mucha más presencia por las tardes, acompañando a niños y niñas a la salida de la escuela. Las observaciones muestran el predominio del sexo masculino en las dos franjas horarias, aunque 
si a la población infantil la consideramos como personas dependientes, o que tiene que ir acompañada, el grupo predominante por las tardes es el femenino. Teniendo en cuenta la población de origen extranjero, su presencia es, en general, minoritaria, pero en todo caso el grupo predominante en esta población es el masculino, con una mayor variabilidad presencial entre hombres y mujeres que en el caso de la población autóctona.

Se observa que la vida en la plaza guarda una clara relación con la división sexual del trabajo, y es un reflejo de una sociedad que hace de las mujeres una fuerza de trabajo doméstico, dedicadas a la atención doméstica y a la vida social del barrio:

«Me encuentro con otras abuelas, pero siempre voy con una chica que es más joven que yo, tiene unos cincuenta o cincuenta y un año, y tiene mellizos y van a la escuela con nuestros niños. Está claro, vamos allí, nos sentamos allí, mirando y charlando y los niños juegan. Vienen algunas abuelas, también se sientan allá y hablamos». [29:19] Rosa, vecina de Ca n'Anglada, 70 años. Nacida en Terrassa. Reside en el barrio desde los 32 años.

Pero a la división sexual del trabajo en los barrios también se añade la edad, ya que hay todo un sector de población, el de las personas adultas, que por motivos laborales tiene una presencia más limitada en las plazas, al contrario que le pasa a la población infantil o las personas mayores. En todo caso, una posible anomalía sería el caso de la juventud, sobre todo la que ya es más independiente, que a pesar de disponer de tiempo, tiene presencias muy limitadas en la plaza, y prefieren espacios más reservados donde haya menos vigilancia adulta y puedan hacer más cómodamente actividades que entrarían en conflicto con otros usuarios en lugares como la Plaza de Ca n'Anglada.

Al mismo tiempo, la presencia de inmigrantes en la plaza responde a un claro modelo de uso compartido del espacio pero no en el tiempo. Es decir, la presencia de población recién llegada, sobre todo de hombres adultos y jóvenes, crece en importancia en momentos en que la presencia de personas autóctonas no es predominante, como bien entrada la tarde, por las noches o los fines de semana, como se pudo observar en algunas visitas que se hizo a la plaza. La interacción, por tanto, tiene dificultades en las plazas, y hay que saber distinguir entre el derecho a no relacionarse del conjunto de personas que visitan una plaza y la discriminación y marginación que se puede ejercer contra un colectivo concreto, como sería el caso comentado de la población de origen extracomunitario que son también vecinos y vecinas del barrio.

\subsection{Las ausencias en la plaza}

Las observaciones realizadas han puesto de manifiesto que algunos colectivos de residentes están prácticamente ausentes de un espacio tan destacado y emblemático para el barrio como es su plaza central. Así, en las tardes de los 
días laborales casi no hay población infantil de origen marroquí, aunque es el momento en que, al salir de la escuela, la plaza es utilizada mayoritariamente por la población infantil y más joven. Es, pues, significativo destacar que mientras que en las escuelas primarias (públicas) la mezcla entre niños y niñas autóctonas y recién llegadas no es nada problemática, al salir de la escuela la separación es visible: la plaza es ocupada por población infantil autóctona con sus madres y abuelas, mientras que la misma población de origen marroquí está ausente. Mientras tanto, jóvenes de origen marroquí se encuentran en espacios segregados cercanos, como el polideportivo, donde la única actividad posible es el fútbol (Díaz-Cortés, 2009). Son espacios impulsados desde el gobierno municipal y que comportan un claro control y la invisibilización de estos chicos en los espacios más emblemáticos del barrio. Por otra parte no encontramos allí a chicas de origen marroquí, las que localizamos en espacios cerrados o no tan concurridos, como la biblioteca del barrio, plazas del interior del barrio o en la casa de alguna de ellas.

De hecho, las mujeres y la población infantil y juvenil de origen marroquí está casi siempre ausente de la plaza y sólo en ocasiones en que no hay allí grupos de personas autóctonas - especialmente cuando no hay madres y abuelas autóctonas -, sobre todo en ciertos momentos del fin de semana, podemos ver a esta población recién llegada utilizando la plaza. También, otras plazas y parques fuera del barrio son aprovechados por estos grupos para distraerse. Aún es más raro ver a chicas de origen marroquí. Aunque hay que decir también que éstas ya tienen en algunos casos una clara actitud de reivindicación de autonomía personal en los espacios públicos, cuestionando la autoridad paterna:

«Sí, como mi padre, me dice de ponerme [el velo] y yo "no me da la gana", no me va a decir "póntelo" a la fuerza, no. Y yo le digo cuando me dé la gana me lo voy a poner, no ahora. Pasa de mí, como paso yo de él. [...] no tienes que obligar a tu hija, "pon, pon, pon"». [38:33] Suad, vecina de Ca n'Anglada, 15 años. Inmigrada recientemente desde Tánger (Marruecos). Vive en el barrio desde los 9 años.

\subsection{Los espacios públicos del barrio: espacios feminizados y etnicizados}

Los espacios públicos del barrio de Ca n'Anglada y, en concreto, su plaza central, responden a un modelo de uso y de presencias y ausencias donde el género y la etnia juegan un papel fundamental: es ante esta constatación que podemos hablar de la feminización y etnicización de estos espacios públicos. En efecto, se observa que hombres y mujeres llevan a cabo diversas tareas en el espacio público según el rol asignado tradicionalmente: el trabajo doméstico no remunerado hace que la mujer de mediana edad y mujeres mayores sean muy presentes en el barrio y sea el grupo predominante en los espacios públicos cuando, aparte de ser mujer, es madre o abuela de población en edad infantil y 
dependiente. El trabajo no doméstico y remunerado hace que el hombre en edad de trabajar quede fuera de la cotidianidad de los barrios aunque los que son padres suelen aprovechar sus ratos libres, sobre todo durante el fin de semana, para acompañar a los niños en los espacios públicos:

«Sí, cuando salen los críos del cole en verano, pues, no los vas a encerrar en casa, los bajas al parque y allí ellos ya están jugando. [Te encuentras] normalmente madres, es muy raro que encontremos algún padre, lo normal son madres que sacamos a los críos al parque». [3:37] Silvia, vecina de Ca n'Anglada, 34 años. Nacida en Córdoba (Andalucía). Reside en el barrio desde los 30 años y desde los 18 en Terrassa.

La importancia de la dimensión étnica también se pone de manifiesto al analizar, sobre todo, las ausencias en los espacios públicos. Se trata de situaciones en que la identidad y los imaginarios colectivos son utilizados por parte de población autóctona para hacer apropiaciones que no son inclusivas, sino exclusivas, de los espacios públicos más emblemáticos y referenciales. En este sentido, las mujeres inmigrantes recién llegadas además de hacer frente a su marginación en el ámbito familiar que antes se ha mencionado, también deben adaptarse a situaciones que implican evitar ciertos espacios y ciertas presencias, algo que se agravó más a raíz de los incidentes de 1999:

«Antes bien, pero ahora no, mi marido coge el coche y vamos fuera...Antes iba sólo con los niños, ahora cuando está lleno de gente y viene gente de aquí... [...], aquí en el barrio hay un parque muy, muy majo, pero damos una vuelta y venimos, pero la mayoría de veces yo, mi marido y los niños vamos a fuera». [19:10] Munira, vecina de Ca n'Anglada, 36 años. Inmigrada recientemente desde Rabat (Marruecos).

\subsection{Recelos ante los nuevos usos y las nuevas presencias en los espacios públicos}

Los incidentes de 1999 son la expresión más clara y extrema de las posturas más intransigentes con el asentamiento de población de origen extranjero en el barrio. Son diversas las versiones que intentan explicar el inicio de los incidentes, pero el hecho que cabe reseñar es la participación de vecinos y vecinas del barrio en las agresiones a población de origen marroquí residente también en Ca n'Anglada:

«Yo estaba en el balcón de mi casa y vi toda la manifestación, no sé muy bien que fue, no sé si es que hicieron algo a una niña, o algo y ya se lió. Pues pensaba "ostras; Qué pasa aquí, qué está pasando?" claro, yo no sabía nada y vi todo el meollo de la gente que subía, y no me metí en la manifestación porque no tengo nada que manifestarme a este respecto, si lo quieren así a mi mientras no me molesten, a mi si me pasara alguna cosa o miembros de mi familia se vie- 
ran...no, porque claro, hay mucha gente que dice "es que a mi me pasó", pero claro no lo puedes meter a todos en el mismo saco porque te pase algo. Hay moros malos como hay españoles malos, eso de porque sean moros, vale que huelen mal, eso es verdad pero mira su olor natural es así, pero nada más". [13:50] Helena, vecina de Ca n'Anglada, 27 años. Nacida en Terrassa. Vive en el barrio desde los 15 años.

En este contexto, espacios públicos como la Plaza de Ca n'Anglada se han transformado en un recurso simbólico e identitario excluyente para algunas personas. En los mismos días que se produjeron los incidentes racistas, los periódicos, locales y estatales, reproducían comentarios muy parciales de vecinos y vecinas autóctonas que ponían de manifiesto el sentimiento de apropiación e identificación hacia la plaza y como percibían negativamente la presencia de los y las recién llegadas:

«Ya lo veis, se quieren follar a nuestras mujeres y la policía los defiende a ellos. Compañeros, acordaos que esa plaza es la Roja y que es nuestra, que allí hemos luchado por todo este barrio. Y al moro que se mee en esta plaza le vamos a cortar la punta del...» (La Vanguardia, 18 de julio de 1999).

«Aquí, ya mismo nosotros seremos los extranjeros y ellos los españoles. De momento, ya se han hecho los amos de la plaza» (Diari de Terrassa, 14 de julio de 1999).

Los estereotipos y el prejuicio que sobre Ca n'Anglada y sobre comunidades de origen marroquí - la más mayoritaria en el barrio - se expresan tanto desde el exterior como desde el interior del barrio, son el caldo de cultivo de brotes violentos como los sucedidos en Terrassa en 1999:

«Pues, inseguridad más bien por la noche, porqué lo único que ves por la calle son inmigrantes. Yo tengo una amiga que vive por allí abajo, más por el centro del barrio, y enfrente hay un bar también de ellos y siempre está por allí lleno y entonces a mi también me da miedo ir allí. No sé, si te ven sola pués, no sé, un poco de miedo sí que tienes». [24:21] Julia, vecina de Ca n'Anglada, 22 años. Vive en el barrio desde su nacimiento.

«A Ca n'Anglada le dicen Marruecos. La gente ya le ha puesto a Ca n'Anglada Marruecos, "ah! vas a Marruecos" "ah! Vives en Marruecos"》. [8:54] Ángeles, vecina de Ca n'Anglada, 35 años. Inmigrada proveniente de Guayaquil (Ecuador). Vive en el barrio desde los 32 años.

En este contexto, las presencias de mujeres autóctonas en espacios públicos emblemáticos, como las situaciones descritas anteriormente, suponen la "normalidad" de la vida cotidiana en plazas y calles de Ca n'Anglada para los sectores vecinales más reacios y opuestos a la nueva realidad social y cultural 
que se vive en el barrio. Se trata de la aplicación de un cierto control de las presencias en los espacios públicos, lo que supone que plazas como la de $C a$ n'Anglada no sean espacios de inclusión y que se haga necesaria la búsqueda de espacios y lugares alternativos para que se pueda dar la inclusión y las relaciones sociales y comunitarias no basadas en la discriminación.

Cabe advertir, que las posturas más cerradas con respecto a la nueva inmigración se pueden encontrar en entidades asociativas muy significativas y relevantes para el barrio, como es el caso de la asociación de vecinos o colectivos de personas mayores:

«El tema de la asociación de vecinos, se quejan de la limpieza por decirte algo [y] a veces se toma el papel de la inmigración como un papel para obligar al ayuntamiento [...]. En Ca n'Anglada hay una concentración de servicios porqué todos tienen miedo de Ca n'Anglada, los proyectos y todos están en Ca n'Anglada, la mayoría, aunque ahora los quieren hacer en otros barrios, pero es verdad que aquí hay más trabajo que en otros barrios». [6:18] Amal, personal técnico municipal no residente en el barrio. Inmigrada recientemente desde Ksar-el-Kbir (Marruecos).

Estas entidades, además, concentran la interlocución barrio-ayuntamiento, aspecto que supone una clara monopolización de "la voz" del barrio y, sobre todo, una clara influencia y condicionamiento sobre las políticas municipales en el barrio. El asentamiento de la inmigración de origen extracomunitario también se utiliza por estas entidades para problematizar la situación del barrio y reclamar la necesidad de inversiones públicas, un enfoque del todo negativo y contraproducente para la cohesión social y comunitaria (Díaz-Cortés, 2009).

\section{ESPACIOS FEMENINOS DE ENCUENTRO}

\section{Espacios públicos emblemáticos aparentemente intocables}

El caso de Ca n'Anglada pone de manifiesto las dificultades de interacción social que se pueden dar en los espacios públicos cuando coexisten diferentes realidades étnicas y culturales. La existencia de una comunidad más o menos homogénea - que se articula a través de una marcada identidad de barrio y arraigo a sus espacios públicos concretos - puede suponer dificultades en la incorporación a los espacios públicos y a la vida pública del barrio de personas recién llegadas de otros contextos culturales, y esto resulta dramáticamente cierto cuando se problematiza la presencia de estas personas en espacios que la comunidad originaria considera emblemáticos del barrio, como por ejemplo la Plaza de Ca n'Anglada. En este contexto relacional, la tradicional preponderancia de la presencia femenina «autóctona» en los espacios públicos emblemáticos del barrio se convierte, de una manera consciente o no, en un mecanismo de control y dominio directo sobre las pautas de uso y apropiación de los espacios públicos 
en la compartimentación horaria y por origen étnico de algunos espacios de encuentro emblemáticos. El paisaje social de los espacios públicos, en particular de la Plaza de Ca n'Anglada, es tan interiorizado y tan cargado de simbolismo que parece que cualquier alteración se convierta en un grave descalabro para determinados sectores de residentes en el barrio.

Para los sectores más reticentes al asentamiento de población de origen extranjero, visualizar un cambio en el paisaje social de los espacios públicos implica casi automáticamente la necesidad de la movilización social de la propia comunidad para detener la transformación social implícita en esta nueva imagen y que, supuestamente, representan una pérdida de valores, de seguridades, de estatus. De esta manera, las mujeres autóctonas, identificadas como las usuarias tradicionales, necesarias y lógicas de los espacios públicos, se convierten en el recurso visual y simbólico de los sectores más reticentes para medir el nivel de cumplimiento de los usos y apropiaciones tradicionales:

«Yo ahí conozco a una persona en el bloque que la abuela sale a custodiarlo para que no se junte... no es porque le pase algo, para que no se junte con el marroquí. Eso lo he visto yo, lo he comprobado, porque yo lo he escuchado lo que le ha dicho al chiquito, y yo digo "que lástima", que lástima, porque lo arruinan, porque le arruinan la mente. [...] ella se para ahí en la esquina y lo vigila, y así el niño no disfruta de la niñez, lo pienso yo. Es un niño y tiene que jugar, yo no puede ir a elegirle a él [...]». [10:41] Raquel, vecina de Ca n'Anglada, 60 años. Inmigrada proveniente de Buenos Aires (Argentina). Vive en el barrio desde los 58 años.

Así, el paisaje social y visual de la Plaza de Ca n'Anglada se convierte en una especie de barómetro excluyente: mientras la imagen visible preponderante en la plaza siga siendo la de mujeres y abuelas autóctonas con sus niños y niñas, la situación es considerada como normal, de acuerdo con el paisaje tradicional. Cuando en este paisaje tradicional se hacen presentes personas y usos inusuales entonces es cuando se movilizan las quejas o las actitudes de rechazo que pueden derivar en violencia.

\section{2. (Micro)espacios públicos de esperanza}

Los espacios de encuentro con menos contenido identitario o simbólico son los que ofrecen, hoy por hoy, los ámbitos más idóneos para que mujeres de uno y otro origen se encuentren e intercambien experiencias. La iniciativa individual de un número significativo de mujeres vinculadas a servicios sociales y comunitarios del municipio o de vecinas del barrio a través del encuentro cotidiano en espacios vinculados a la actividad diaria que desarrollan estas mujeres en $C a$ n'Anglada, como las Asociaciones de Madres y Padres de Alumnado (AMPA), con incidencia directa en las escuelas o actividades destinadas a las mujeres o espacios asociativos de mujeres que se ubican en el centro cívico u otros equipamientos municipales, son ámbitos donde se expresa con mayor normalidad la 
relación e interacción entre personas de diferentes orígenes culturales, es decir, la función original del espacio público:

« [...] es una iniciativa muy personal de cada uno. Es iniciativa del educador que está en Distrito Joven, es iniciativa de la educadora de Servicios Sociales pero lo que es desde la política no...». [28:33] Núria, vecina de Ca n'Anglada, 24 años, nacida en Terrassa. Vive en el barrio desde su nacimiento.

«Y nosotras estamos hartas de estar en el colegio con ellos y ellos con nosotros, y aquí [en la Escuela de Madres] nosotras no tenemos ningún problema de convivencia». [3:63] Gloria, vecina de Ca n'Anglada, 37 años. Inmigrada proveniente de Puertollano (Castilla-La Mancha). Vive en el barrio desde los 25 años.

«Tenemos un proyecto que es la Escuela de Madres, l'Escola Pont [de Mares Magribines]. Hemos intentado, ha ido muy bien el proyecto, hacíamos clases de lengua y charlas informativas, cosas de formación e información sobre la cultura, sobre los recursos, y así hemos hecho salidas a fomento, bueno a los recursos que tenemos en la ciudad. Fue muy bien, y hay algunas que ya trabajan, hemos hecho cursos de cocina, muchas que han espabilado. Quiero decir que estas mujeres como todas tienen ganas de avanzar, de participar. Trabajan algunas en restaurantes, en Fomento con planes de ayuda, hay otras que hacen trabajo de confección en casa, bueno van haciendo poco a poco. Para ellas es mejor que nada...El proyecto de la Escola Pont es otro [...]. La concejala sí que tiene mucho interés en trabajar el tema de la mujer y fomentar el papel de la mujer inmigrada...». [6:10] Amal, personal técnico municipal no residente en el barrio. Inmigrada recientemente desde Ksar-el-Kbir (Marruecos).

Estos espacios de encuentro que no son lo que tradicionalmente se ha entendido como espacios públicos son los que, hoy por hoy, resultan más idóneos para que mujeres de uno y otro origen se encuentren, interactúen, intercambien experiencias y, sobre todo, hagan del encuentro cotidiano el mejor mecanismo para combatir los recelos y abrir puentes de comprensión de realidades diferentes a la propia. Las personas usuarias son, sobre todo, mujeres que se localizan en ámbitos menos públicos y con menos influencia política y claramente feminizados. Están relacionados con el ocio (talleres de manualidades, confección, culinarios o de relajación) o con la formación (escuelas públicas, escuela de adultos o asociaciones de madres y padres de alumnado), sin olvidar la existencia de un tejido asociativo que, con menos influencia en la interlocución barrio-ayuntamiento, ejerce una función encomiable desde el punto de vista de la cohesión comunitaria y la creación de dinámicas y espacios inclusivos. Se trata de lugares que rompen con la visión tradicional de espacio público, más vinculados a la propia dinámica social y territorial de la cotidianidad, y que suponen nuevos planteamientos en relación a la idea de espacio público que se están exponiendo desde experiencias y trayectorias diversas (Díaz-Cortés, 2009; Muxí, 2009; Vaiou y Kalandides, 2009; Bühler, 
2009).

Se trata de "microespacios" y dinámicas cotidianas que se contraponen a los espacios más emblemáticos e influyentes que encarnan el posicionamiento más cerrado al cambio social y donde el papel de mujeres es protagonista y destacado, sobre todo debido a que están vinculados a la proximidad y a la cotidianidad del barrio, un ámbito donde las mujeres tienen una presencia mayoritaria.

«[Silvia] Yo creo que pasará como en todos los grandes movimientos migratorios, al principio llegan y parecen que te invadan, que estás en contra de su cultura, con los años pues nos acostumbraremos a su cultura igual que ellos se habrán acostumbrado a la nuestra. [Adela] Exactamente. [Gloria] En el momento que los niños que vienen al colegio empiezan a subir pues ya son iguales. [Silvia] Pasó igual cuando llegaron en plan masivo, yo te cuento porque soy de Córdoba, cuando llegaron los andaluces aquí pasó igual, pues como que traíamos nuestras costumbres, traíamos una forma de ver la vida distinta a como se vivía aquí, y todo el mundo pensaba "ostia, los andaluces, apartaros de ellos" ahora prácticamente estamos mezclados, y llegará un punto que pasará igual». [3:71] Adela, vecina de Ca n'Anglada, 33 años. Nacida en Terrassa. Vive en el barrio desde su nacimiento; Gloria, vecina de Ca n'Anglada, 37 años. Inmigrada proveniente de Puertollano (Castilla-La Mancha). Vive en el barrio desde los 25 años; Silvia, vecina de $\mathrm{Ca}$ n'Anglada, 34 años. Nacida en Córdoba (Andalucía). Reside en el barrio desde los 30 años y desde los 18 en Terrassa.

Son microespacios y microdinámicas que intentan romper con el sobrecontrol y la sobreapropiación que sectores del barrio hacen de espacios y ámbitos de la vida cotidiana. Hoy por hoy, estos son los espacios de encuentro de esperanza del barrio, la esperanza de que las mujeres del barrio vuelvan a tener un papel clave en el avance comunitario, con autonomía y autoorganización, la esperanza de que el conjunto del barrio supere los recelos y el rechazo al cambio social y cultural.

\section{CONCLUSIONES Y PROPUESTAS: REDEFINIR EL ESPACIO PÚBLICO PARA FORTALECER EL PAPEL DE LA MUJER EN LOS BARRIOS.}

El estudio de Ca n'Anglada pone de manifiesto las dificultades de interacción social que se pueden dar en espacios públicos tradicionales y emblemáticos, por razones de género y por razones culturales. Pero si la cotidianidad muestra esta realidad, también despuntan realidades más esperanzadoras que hemos querido identificar con la existencia de microespacios relacionales, la mayoría de ellos en ámbitos cerrados pero donde se desarrolla una intensa sociabilidad. Así pues, hay que reformular la idea de espacio público, pues si no se supera la tradicional división entre espacio público y espacio privado - una división que es constantemente desbordada en la cotidianidad de los barrios - se seguirá 
errando en los análisis y en las propuestas para la superación de las discriminaciones por razones de género. Cuando decimos repensar la idea de espacio público hacemos referencia a la necesidad de vincularla a lo que la realidad social muestra cotidianamente: que las personas se relacionan e interaccionan en el transcurso de su jornada diaria en diferentes tipos de espacios y situaciones, siendo la idea de espacios de vida cotidiana, el marco de análisis más apropiado para comprender mejor la situacionalidad que la propia vida urbana implica y, también, para desarrollar políticas públicas en el barrio. Finalmente, hay que reivindicar el papel de la mujer como motor de cambio en los ámbitos urbanos y sociales donde su presencia es habitual. Ello implica, por un lado, reconocer que la desigualdad y la discriminación continúan siendo una realidad que condiciona la presencia de las mujeres en los barrios, (un aspecto que parece haberse "normalizado" e incluso invisibilizado, sobre todo a nivel institucional). Este primer reconocimiento previo y necesario tiene que ir acompañado de un valiente proceso en que las mujeres que tengan una vinculación directa y cotidiana en los barrios sean agentes activos y decisivos en las intervenciones sociales y urbanísticas que se desarrollen, fortaleciendo, en definitiva, el papel político de las mujeres en el ámbito más próximo y cotidiano.

\section{AGRADECIMIENTOS}

Este artículo se sitúa en una línea de investigación más amplia del Grup de Recerca de Geografia i Gènere del Departament de Geografia de la Universitat Autònoma de Barcelona y que ha estado financiada por el Ministerio de Educación en los últimos años (SEJ2006-09837, CSO2009-10913) y la generalitat de Catalunya (SGR-2009-1321).

\section{BIBLIOGRAFÍA}

Ajuntament de Terrassa (2007) Anuari Estadistic de Terrassa 2007. Foment de Terrassa y Ajuntament de Terrassa, Terrassa.

Albet A, Clua A; Díaz-Cortés F (2006) Resistencias urbanas y conflictos creativos: lo público como espacio de reconocimiento. In Nogué J y Romero J (eds.) Las otras geografías. Tirant lo Blanch, València: 405-423.

Baylina M (2004) Metodología para el estudio de las mujeres y la sociedad rural. Estudios Geográficos, 65 (254): 5-28.

Benach N, Tello R (2004) En los intersticios de la renovación: estrategias de transformación del espacio y flujos de población en Barcelona. Revista de Geografía, 3: 93-113.

Bondi L (1998) Gender, class, and urban space: public and private space in contemporary urban landscapes. Urban geography, 19(2): 160-185.

Bondi L, Domosh M (1998) On the contours of public space: a tale of three women. Antipode, 30(3): 270-289.

Borja J (2003) La ciudad conquistada. Alianza Editorial, Madrid.

Borja J (ed.) (1995) Barcelona: un modelo de transformación urbana, 1980-1995. Programa de Gestion Urbana (PGU-LAC), Quito.

Borja J, Muxí Z (2001) El espacio público: ciudad y ciudadanía. Diputació de Barcelona, Barcelona. 
Bühler E (2009) Public spaces and social diversity. Editorial to the special issue. Geographica Helvetica, 1: 2-4.

Busquets J (2004) Barcelona. La construcción urbanística de una ciudad compacta. Ediciones del Serbal, Barcelona.

Chelkooff G, Thibaud JP (1992-1993) L'espace public, modes sensibles. Les Annales de la Recherche Urbaine, 57-58: 7-16.

Corbridge S, Thrift N J, Martin R (1994) Money, power and space. Basil Blackwell, Oxford.

Crang M (2005) Qualitative methods: there is nothing outside the text? Progress in Human Geography, 29(2): 225-233.

Crang M (2002) Qualitative methods: the new ortodoxy? Progress in Human Geography, 26(5): 647-655.

Davies G,Dwyer C (2007) Qualitatives methods: are you enchanted or are you alienated? Progress and Human Geography, 31(2): 257-266.

Davis M (2001) Control urbano, la ecología del miedo. Más allá de Blade Runner. Virus, Barcelona.

Díaz-Cortés F (2009) Espai públic, vida cuotidiana i identitat de barri a Terrassa: la construcción material $i$ social de Can Palet $i$ Ca n'Anglada a través d'una geografia de la proximitat. Tesis Doctoral, Universitat Autònoma de Barcelona, Bellaterra.

Díaz-Cortés F (2006) Los equipamientos en la ciudad. Conciliación del trabajo productivo, del trabajo reproductivo y del tiempo libre en los barrios. In DeL Caz R y Rodríguez M (eds.) El derecho a los equipamientos, a los edificios públicos. Informe Valladolid 2006. Escuela Técnica Superior de Arquitectura de Valladolid, Valladolid: 63-66.

Driver F (2003) On geography as a visual discipline. Antipode, 35(2): 227-231.

Ekinsmyth C (2002) Feminist methodology. In Shurmer-Smith P (ed.) Doing cultural geography. Sage Publications, London: 177-185.

Fernández Durán R (1996) La explosión del desorden. La metrópoli como espacio de crisis global, Fundamentos, Madrid.

Flahault E (2001) L'appropiation des espaces chez les femmes seules. Villes en parallele, 32-34: 199-206.

Francis M (1989) Control as a dimension of public space quality. In Altman I y Zube EH (eds.) Public Places and Spaces, Plenum Press, New York: 147-172.

Franck K A, Paxson L (1989) Women and urban public space. In Altman I y Zube EH (eds.) Public Places and Spaces. Plenum Press, New York: 121-146.

García Ballesteros A (1998) Métodos y técnicas cualitativas de investigación en geografía social. In García Ballesteros A (coord.) Métodos y técnicas cualitativas en geografía social, Oikos-Tau, Barcelona: 13-26.

García Ballesteros A (1982) El papel de la mujer en el desarrollo de la geografía. In Durán MA (ed.) Liberación y Utopía, Akal Universitaria, Madrid: 119-141.

Garcia Ramon M D (2008) Espacios asexuados o masculinidades y feminidades espaciales? Hacia una geografía del género. Semata, Ciencias Sociais e Humanidades, 20: 25-51.

Gleson B J (1995) A public space for women: the case of charity in colonial Melbourne. Area, 27(3): 193-207.

Greed C H (ed.) (1996) Investigating town planning. Changing perspectives and agendas. Addison Wesley Longman, Harlow.

Greed C H (1994) Women and planning: creating gendered realities. Routledge, London.

Hayden D (1995) The power of place. Urban landscapes as public history. MIT Press, Cambridge.

Hayden D (1981) What would a non-sexist city be like? Speculations on housing, urban design, and human work. Signs, 5 (3): 170-187.

Jackson P (1989) Domesticating the street: the contested spaces of the high street and the mall. In Fyfe N (ed.) Images of the street: planning, identity and control in public space, Routledge, London: 176-191.

Katz C (1994) Playing in the field. Professional Geographer, 46(1): 67-72.

Kern L (2005) In place and at home in the city: connecting privilege, safety and belonging of women in Toronto. Gender, Place and Culture, 12(3): 357-377. 
Lacueva J L, Márquez M, Plans L (2007) Combat per la llibertat. Memòria de la lluita antifranquista a Terrassa (1939-1979). Fundació Torre del Palau, Terrassa.

López de Lucio R (2000) El espacio público en la ciudad europea: entre la crisis y las iniciativas de recuperación. Implicaciones para Latinoamerica. Revista de Occidental, 230-231: 105-121.

McDowell L (2000) Género, identidad y lugar: un estudio de las geografias feministas, Cátedra, Madrid.

McDowell L (1992) Multiple voices-speaking from inside and outside the project. Antipode, 24: $56-72$.

Mitchell D (2003) The right to the city. Social justice and the fight for public space. The Guilford Press, New York-London.

Montaner J M (2004) La evolución del modelo Barcelona (1979-2002). In Borja J y Muxí Z (eds.) Urbanismo en el siglo XXI: una visión crítica. Bilbao, Madrid, Valencia, Barcelona. Edicions UPC, ETSAB (Arquitext), Barcelona: 203-219.

Muxí Z (2009) Barris per a viure. Nous Horitzons, 195: 82-89.

Pain R (1997) Social geographies of women's fear of crime. Transactions of the Institute of British Geographers, 22: 231-244.

Pain R, Townshend T (2002) A safer city centre for all? Senses of 'community safety' in Newcastle upon Tyne. Geoforum, 33(1): 105-119.

Prats M (1998) Gènere, ús del temps i geografia. Documents d'Anàlisi Geogràfica, 32: 175-188.

Prats M (1997) El temps de la vida quotidiana de les dones de la ciutat de Barcelona. Tesis Doctoral, Universitat Autònoma de Barcelona, Bellaterra.

Riley M; Harvey D (2007) Talking geography: on oral history and the practice of geography. Social \& Cultural Geography, 8(3): 345-351.

Roca J (ed.) (1994) El futur de les perifèries urbanes. Canvi econòmic i crisi social a les metròpolis contemporànies. Institut de Batxillerat "Barri Besòs", Barcelona.

Rogers A (1998) Los espacios del multiculturalismo y la ciudadanía. Revista Internacional de Ciencias Sociales, 156. (http://www.unesco.org/issj/rics156/rogerspa.html) [Consultada el 15 de octubre de 2009].

Ruddick S M (1996) Constructing difference in public spaces: race, class and gender as interlocking systems. Urban Geography, 17(2): 132-151.

Sandercock L, Forsyth A (2000) A gender agenda: new directions for Planning theory. Journal of the American Planning Association, 58(1): 49-59.

Sennet R (1978) El declive del hombre público. Edicions 62, Barcelona.

Taylor S J, Bogdan R (1996) Introducción a los métodos cualitativos de investigación. La búsqueda de significados. Paidós, Barcelona.

Tello R, Quiroz H (2009) Ciudad y diferencia. Género, cotidianeidad y alternativas. Ediciones Bellaterra, Barcelona.

Vaiou D (2010) Gender, migration and socio-spatial transformation in Southern European cities. In Pike A, Rodríguez-Pose A y Tomaney J (eds.) Handbook of local and regional development. Routledge, London (en prensa).

Vaiou D, Kalandides A (2009) Cities of "others": public space and everyday practices. Geographica Helvetica, 1: 11-20.

Valentine G (1989) The geography of women's fear. Area, 21: 385-390.

Valle T (1997) Andamios para una nueva ciudad. Lecturas desde la antropología. Cátedra, Madrid.

Walzer M (1986) Pleasures and costs of urbanity. Dissent, 33(4): 470-475.

Wekerle G (1984) A woman's place is in the city. Antipode, 16(3): 11-19.

Wilson E (1995) The invisible flâneur. In Watson S, Gibson K (eds.) Postmodern cities and spaces. Blackwell, Oxford: 59-79. 